\title{
Non-traumatic myelopathy associated with prolonged hyperextension during swimming: an unusual variation of Surfer's Myelopathy
}

\author{
Edward Green ${ }^{1} \cdot$ Umme Sara Zishan $^{2} \cdot$ Nicola Robertson $^{3} \cdot$ Joseph Papanikitas $^{2} \cdot$ Sarah Yanny $^{2} \cdot$ Richard Hughes $^{2} \cdot$ \\ David McKean ${ }^{2}$
}

Received: 8 November 2017 / Revised: 9 February 2018 / Accepted: 9 February 2018

(c) International Spinal Cord Society 2018

\begin{abstract}
Introduction Surfer's myelopathy (SM) is a rare cause on non-traumatic acute myelopathy. This has classically been described in novice surfers however has been reported in other scenarios.

Case presentation We present a case of non-traumatic paraparesis associated with prolonged cervical hyperextension during swimming with imaging characteristics consistent with an unusual variation of SM in a swimmer.

Discussion SM and its variants should be considered in the differential diagnosis of any patient presenting with an anterior spinal cord syndrome with a history of either sustained or repeated spinal hyperextension.
\end{abstract}

\section{Introduction}

Surfer's myelopathy (SM) is a rare cause of acute myelopathy without traumatic association, most commonly described in novice surfers. Initially labelled by Thompson et al. in 2004 [1], there have been multiple case reports and case series since, with a common theme of affected individuals lying prone on surfboards with spinal hyperextension leading to onset of back pain and progressive neurological deficits.

Despite the precise pathogenesis of SM being unknown and multiple processes being put forward, it is universally agreed that the mechanism is vascular in origin. However, no study has provided angiographic evidence of vascular compromise in a patient with SM [2].

Imaging characteristics are relatively consistent throughout the literature, with magnetic resonance imaging (MRI) demonstrating swelling and central high T2 signal

Edward Green

edd_green@hotmail.com

1 Oxford University Hospitals NHS Trust, Windmill Road, Headington, Oxford, UK

2 Stoke Mandeville Hospital, Buckinghamshire Healthcare NHS Trust, Aylesbury, UK

3 The Royal Free NHS Trust, Pond Street, London, UK extending longitudinally over the affected cord segment, usually the mid-thoracic region to the conus medullaris [3]. Restricted diffusion on diffusion weighted imaging (DWI) and apparent diffusion coefficient (ADC) sequences, indicating cord infarction, is found in most but not all cases that have been identified. Contrast enhancement is not a feature of this condition [2].

Treatment protocols that have been recommended are based on early steroid therapy, induced hypertension, and early commencement of physical rehabilitation with transfer to a specialist spinal rehabilitation unit for those cases with refractory symptomatology [2]. Oral or intravenous anticoagulation has been suggested as a treatment due to the presumed ischaemic mechanism of spinal cord damage, although this has not been fully established into treatment protocols.

Clinical outcomes from SM vary greatly, from complete recovery to ongoing paraplegia. The patient's prognosis appears to be predicted most accurately by the severity of neurological deficit at nadir (usually at presentation), and patients presenting with ASIA scores of A or B demonstrate little or no improvement in their condition on follow-up [3]. Prognosis does not however appear to be linked to the initial MR imaging characteristics [3].

We present a case of non-traumatic paraparesis associated with prolonged cervical hyperextension during swimming with imaging characteristics consistent with an unusual variation of SM. 
Fig 1 Sagittal CT (a) and axial T2 (b), sagittal T2 (c), and sagittal DWI (d) MRI at presentation. CT demonstrates no acute cervical spine fracture, with maintenance of normal vertebral alignment. MRI demonstrates C5-6 and C6-7 degenerative disc-osteophyte bars, with central cord high $\mathrm{T} 2$ signal and corresponding restricted diffusion
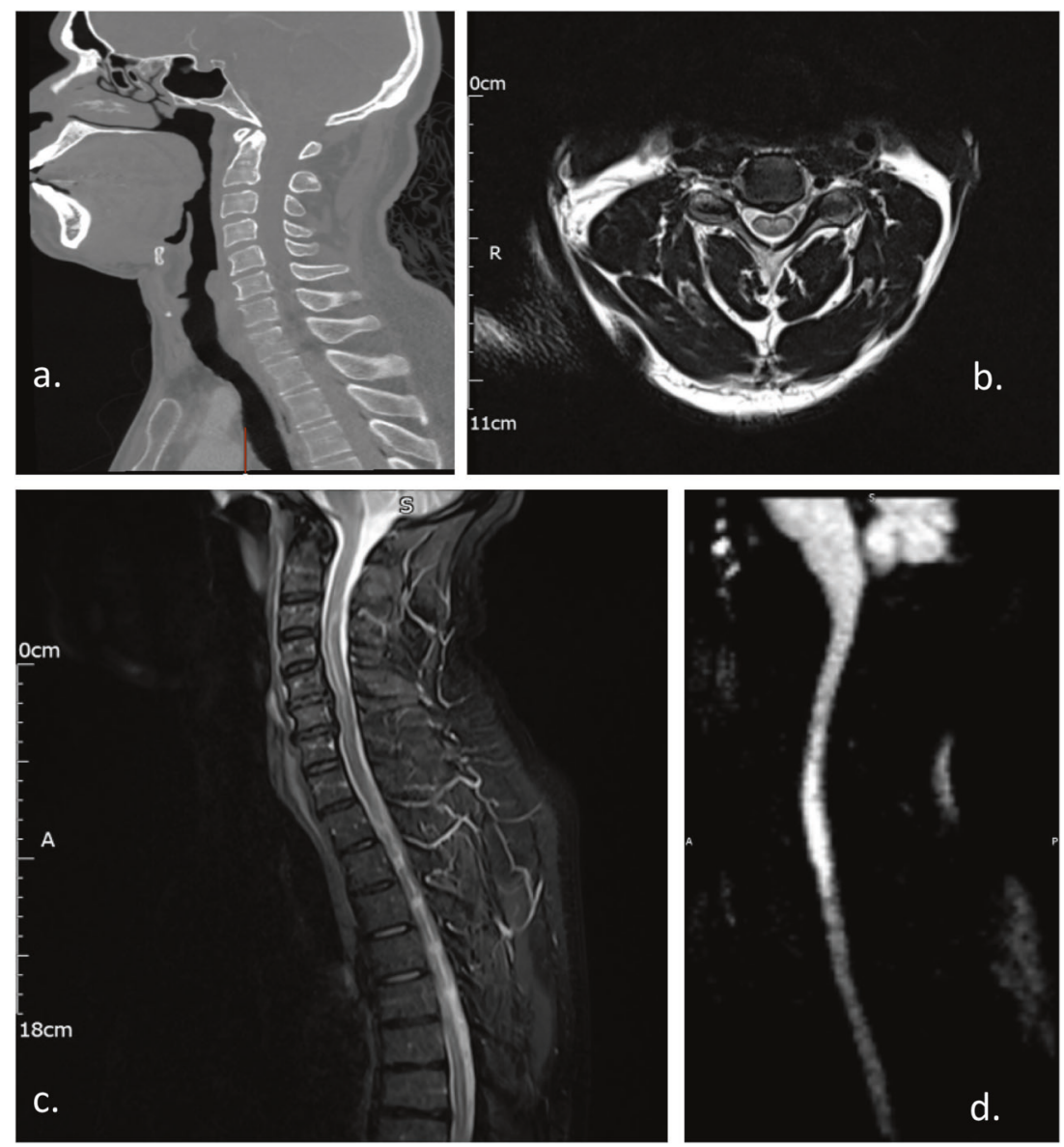

\section{Case presentation}

A 55 year-old male patient with no significant past medical history presented to the Emergency Department after developing neurological symptoms whilst swimming. A previously regular swimmer, he had not swum for approximately 2 years before this session. Twenty minutes into the swim, using the breaststroke technique, he developed sudden onset neck and upper back pain radiating down his right arm. On leaving the water to sit down, his symptoms progressed to bilateral upper limb paraesthesia followed by weakness and paralysis, with subsequent lower limb involvement also. At his nadir, the patient had complete paralysis of all four limbs, with co-existent bowel and bladder dysfunction. A detailed clinical history was taken and there was no history of previous minor neck injury, traumatic fall or motor vehicle accident. No further relevant medical history was recorded.

Initial MRI (Fig. 1) on presentation demonstrated central linear high $\mathrm{T} 2$ signal in the cervical spine from $\mathrm{C} 3$ to $\mathrm{C} 7$, with a corresponding area of restricted diffusion on DWI sequences. There were minor degenerative disc-osteophyte bar complexes at the level of C5-6 and C6-7. No abnormalities were demonstrated in the thoracic or lumbar cord down to the conus.

A repeat MRI scan 9 days later (Fig. 2) demonstrated persistent central high T2 signal in the expanded cervical spine, with the characteristic 'Owl's eyes' appearance on the axial T2 sequence consistent with spinal cord infarction. DWI sequences again indicated restricted diffusion in the affected cord segment, in keeping with infarction.

A CT of the aortic arch and cervical vessels was performed and no vascular pathology was identified. Normal appearances of the carotid and vertebral arteries were demonstrated bilaterally with no features of dissection or stenosis. Cardiac investigations were normal with no dysrhythmia. Coagulation profile was within the normal range.

\section{Discussion}

Cases of non-traumatic spinal cord ischaemia have been described in surgical patients with an intra-operative hyperlordotic posture [4], demolition workers [5], cheerleaders [6], acute spinal hyperextension [7], and clearly 

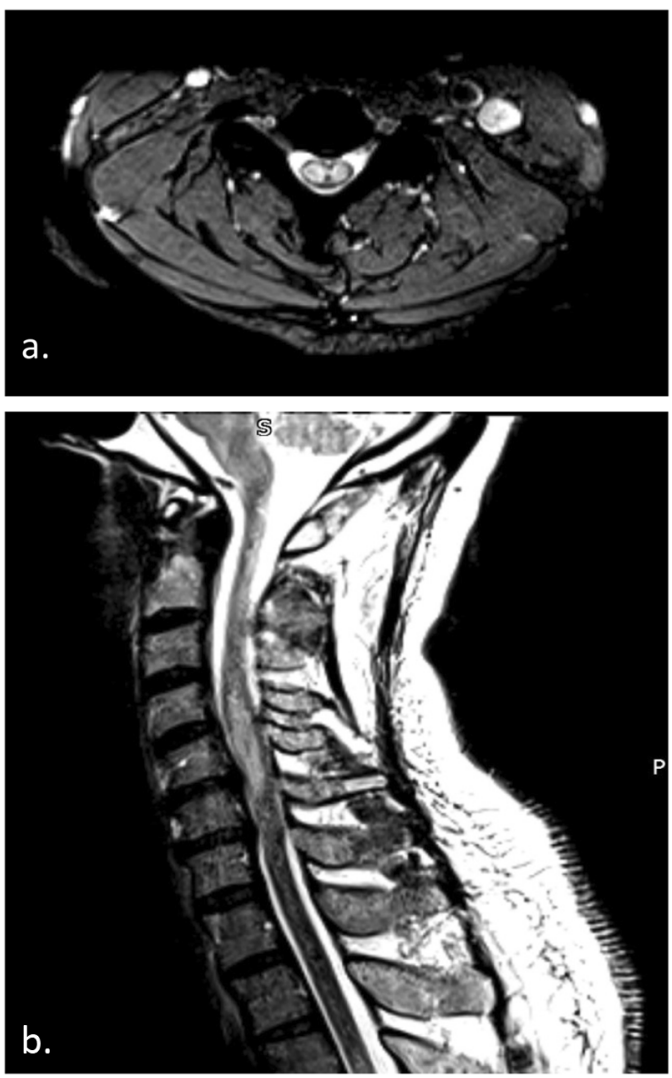

Fig 2 Axial (a) and sagittal (b) T2 signal MRI at 9 days. Central high T2 signal in the affected cord segment-the 'owl's eyes' appearance

(usually novice) surfers [1, 8], but never before in swimmers.

A variety of swimming strokes, particularly breaststroke and butterfly, involve the spinal hyperextension identified as the principle causative mechanism in SM. There is hyperextension of the upper spine during the recovery (inspiratory) phase of the breaststroke cycle, just before the arms are thrown forwards in the thrust phase of the stroke. A prolonged period of breaststroke swimming causes repeated hyperextension of the cervico-thoracic spine, and in our case, led to the development of neurological symptoms.

The most commonly proposed mechanism for the pathogenesis of SM is ischaemia in the anterior spinal cord watershed zone, secondary to either arterial compression, vasospasm, thrombosis, or avulsion [9], secondary to a sustained hyperextension of the spine during lying prone on the surf board. The process by which vertebral hyperextension leads to spinal cord ischaemia has been proposed to be a combination of increased tension on the spinal cord vasculature causing perforator vessel avulsion and subsequent vasospasm, or transient hypoperfusion of watershed zones due to spinal cord tension.

Alternative mechanisms that have been proposed include increased retrograde venous pressure due to inferior vena cava compression and Valsalva leading to venous ischaemia of the cord, or that retrograde embolization of fibrocartilaginous disc material into the sulcal or radicular vessels leads to cord ischaemia and infarction [9].

Prolonged repeated hyperextension of the spine, as in the case of breaststroke swimming, is proposed to have a similar effect on the spinal cord arterial system as is sustained but static hyperextension. Whether the Valsalva manoeuvre that occurs during swimming also contributes to the mechanism of spinal cord ischaemia is open to debate, as is the involvement of the relative hypoxaemia associated with cardiovascular exercise.

Imaging characteristics for patients with SM have been relatively consistent throughout the literature. MRI demonstrates a segment of increased $\mathrm{T} 2$ hyperintensity over the affected levels, usually extending to the conus. Restricted diffusion on DWI and ADC sequences, and a lack of enhancement on administration of contrast are also typical [3]. It is these imaging findings, along with a characteristic non-traumatic history of sustained or repeated spinal hyperextension, that allows SM to be identified over the differential diagnoses. These include acute transverse myelitis, traumatic spinal cord infarction, multiple sclerosis, coagulopathy, intramedullary neoplasms, systemic hypotension, and aortic or vertebral artery dissection $[3,9,10]$. Interestingly, there has been no proven link as yet between the MRI findings at presentation of patients with SM and the severity of the neurological deficit or the degree of neurological recovery [3].

Previous case reports and series have reported age-ranges for affected individuals between 7 [6] and 51 [5]. Our patient is therefore the oldest reported case of SM, and the only reported with pre-existing vertebral pathology. The lower cervical disc-osteophyte bar complexes demonstrated on initial imaging are likely to have predisposed our patient to cervical spinal cord pathology in the context of vertebral hyperextension, and may explain why the affected cord segment in this case was in the cervical spine, rather than thoraco-lumbar spine as has been previously described in the SM literature.

\section{Conclusion}

SM is a non-traumatic cause of acute myelopathy, commonly affecting previously healthy and often young individuals. It should be considered in the differential diagnosis of any patient presenting with an anterior spinal cord syndrome with a history of either sustained or repeated spinal hyperextension. While it has been an uncommon diagnosis in most parts of the world since first being described, increasing awareness of the condition has led to it being recognised in a wider range of clinical settings and patient types. By drawing attention to SM in a 
new clinical context, we aim to increase the understanding of the condition, something that is essential to enable early recognition and treatment, thereby minimising morbidity and maximising the chances of full neurological recovery.

\section{Compliance with ethical standards}

Conflict of interest The authors declare that they have no conflict of interest.

\section{References}

1. Thompson TP, Pearce J, Chang G, Madamba J. Surfer's myelopathy. Spine. 2004;29:E353-356.

2. Freedman BA, Malone DG, Rasmussen PA, Cage JM, Benzel EC. Surfer's myelopathy: a rare form of spinal cord infarction in novice surfers: a systematic review. Neurosurgery. 2016;78:601-11.
3. Nakamoto BK, Siu AM, Hashiba KA, Sinclair BT, Baker BJ, Gerber MS, et al. Surger's myelopathy: a radiologic study of 23 cases. Am J Neuroradiol. 2013;34:2393-8.

4. Roberts DR, Roe J, Baudouin C. Hyperlordosis as a possible factor in the development of spinal cord infarction. $\mathrm{Br} \mathrm{J}$ Anaesth. 2003;90:797-800.

5. Maharaj MM, Phan K, Hariswamy S, Rao PJ. Surfer's myelopathy: a rare presentation in a non-surfing setting and review of the literature. J Spine Surg. 2016;2:222-6.

6. Wadia S, Padmanabhan P, Moeller K, Rominger A. Pediatric surfer's myelopathy. J Emerg Med. 2015;49:e143-145.

7. Albuja AC, Qaiser S, Lightner DD, Raslau FD, Zafar MS, Bernard PA, Baumann RJ. Surfer's myelopathy without surfing: a report of two pediatric patients. Spinal Cord Ser Cases. 2017;3:17008.

8. Steinfeld Y, Keren Y, Haddad E. Spinal cord injury with central cord syndrome from surfing. Spinal Cord Ser Cases. 2018;4:11 https://doi.org/10.1038/s41394-018-0041-3

9. Shuster A, Franchetto A. Surfer's myelopathy - an unusual cause of acute spinal cord ischemia: a case report and review of the literature. Emerg Radiol. 2011;18:57-60.

10. Teixera S, Moser F, Kotton RH. Imaging features and differentials in surfer's myelopathy: a case report. Emerg Radiol. 2016;23:89-92. 